\title{
Gradhiva
}

GRADHI

Revue d'anthropologie et d'histoire des arts

$4 \mid 2006$

Le commerce des cultures

\section{Bernard müller, La Tradition mise en jeu. Une anthropologie du théâtre yoruba}

La Courneuve, éditions Aux lieux d'être, « Mondes contemporains », 2006, 176 p.

Thierry Bonnot

\section{(2) OpenEdition}

Journals

Édition électronique

URL : http://journals.openedition.org/gradhiva/558

DOI : $10.4000 /$ gradhiva.558

ISSN : 1760-849X

Éditeur

Musée du quai Branly Jacques Chirac

Édition imprimée

Date de publication : 1 novembre 2006

Pagination : 130-132

ISBN : 2-915133-44-1

ISSN : 0764-8928

Référence électronique

Thierry Bonnot, « Bernard müller, La Tradition mise en jeu. Une anthropologie du théâtre yoruba », Gradhiva [En ligne], 4 | 2006, mis en ligne le 10 décembre 2008, consulté le 21 septembre 2020. URL http://journals.openedition.org/gradhiva/558 ; DOI : https://doi.org/10.4000/gradhiva.558

Ce document a été généré automatiquement le 21 septembre 2020.

(c) musée du quai Branly 


\title{
Bernard müller, La Tradition mise en jeu. Une anthropologie du théâtre
} yoruba

\author{
La Courneuve, éditions Aux lieux d'être, « Mondes contemporains ", \\ 2006, 176 p.
}

Thierry Bonnot

\section{RÉFÉRENCE}

Bernard müller, La Tradition mise en jeu. Une anthropologie du théâtre yoruba, La

Courneuve, éditions Aux lieux d'être, « Mondes contemporains », 2006, 176 p.

Cet ouvrage retrace l'expérience d'un ethnologue parti de son propre aveu «à la recherche d'un théâtre authentique » (p. 24), le Yoruba Theatre, pour s'apercevoir au final que ce qu'il cherchait n'existait plus et reconnaître qu'il était " arrivé trop tard » (p. 159). Nous pourrions arrêter là ce compte rendu, et ranger cet opuscule aux côtés de tous ceux des ethnologues déplorant la disparition des traditions véritables, la perte de la pureté autochtone et le danger encouru par les rituels ancestraux menacés par la modernité. Mais, heureusement, Bernard Müller ne s'est pas arrêté à ce qu'il n'hésite pas à qualifier d'échec, puisqu'il a consacré sa thèse de l'EHESS ${ }^{1}$ à ce sujet et qu'il en tire aujourd'hui ce livre. La genèse et l'histoire du théâtre yoruba - ou Yoruba Folk Opera -, mêlent différentes influences géographiques et culturelles, avec une forte inspiration religieuse. Son apogée se situe entre 1950 et la fin des années 1970, dans la mouvance de compagnies actives et dynamiques de Travelling Theatre parcourant le Nigeria. Le public était constitué principalement par la classe moyenne supérieure générée alors par la croissance économique du pays. Parmi les "faiseurs de théâtre » - auteurs et producteurs, ici rassemblés dans le "champ des pratiques spectaculaires », ce que Howard Becker aurait appelé un «monde de l'art ${ }^{2}$ »- quelques grandes figures émergent, dont les biographies sont utilement retracées ${ }^{3}$. 
2 Mais la tentative de faire de ce théâtre ambulant un spectacle populaire fut au final un échec. Ses producteurs et acteurs se sont progressivement orientés dans les années 1980 vers la télévision et la vidéo domestique, nouveau mode d'expression fort prolifique traitant les mêmes matériaux narratifs. Ne subsiste aujourd'hui, pour ce qui est du théâtre dans sa forme "classique", qu'un noyau dur se produisant principalement dans des centres culturels, et qui subit une crise profonde. Quant aux textes des pièces, ils ne sont diffusés que de manière restreinte, sous la forme artisanale de tapuscrits photocopiés qui sont lus publiquement ou discutés lors de débats universitaires.

Le projet initial de l'auteur consistait donc à saisir les transformations de la société yoruba du Nigeria à travers une forme théâtrale : mais une fois sur le terrain, observant que ce qui l'intéressait était un objet hétéroclite, "coexistence dynamique " de différentes formes de pratiques spectaculaires, il s'est très vite aperçu qu'il n'arriverait à rien de probant sans mettre à mal nombre d'idées reçues et de présupposés culturalistes. La pièce yoruba étudiée par Bernard Müller est intitulée Odu-Ifà ; sa trame est calquée sur une cérémonie de l'oracle d'Ifà, cérémonie rituelle habituellement privée et individuelle visant à identifier une attaque en sorcellerie afin de mettre en place une stratégie de réparation ou de rétorsion. Contrairement à ce qui se passe dans une cérémonie "réelle ", au cours de la pièce apparaissent en chair et en os les dieux (orisha), les âmes des défunts et autres entités spirituelles dans le cadre de leurs négociations. La description du déroulement de la pièce est particulièrement vivante, et n'instaure aucune rupture artificielle entre acteurs et spectateurs qui participent du même événement. La finesse de la description est d'autant plus méritoire que l'intrigue se joue sur deux échelles, le temps des événements terrestres et le monde du divin (orun en langue yoruba) où se déroule le combat entre sorcière et orisha. Soit deux univers fictionnels dotés de systèmes de références dont le public connaît à l'avance les ressorts, et dont la réminiscence des caractères particuliers fait partie des effets, des émotions attendues par le public, gages d'une représentation réussie.

À la tension dramatique de la pièce correspond la tension d'une consultation réelle d'un oracle. La survenue d'une transe, sur scène ou dans le public, est caractéristique de ce que l'auteur appelle la "confusion des genres ", enchevêtrant un ensemble de scènes de la vie nigériane avec une cérémonie rituelle. "À l'instant où une transe surgit sur une scène de théatre, les cadres de convention qui se distinguent habituellement se confondent subrepticement.»(p.80) D'où la difficulté de travailler à partir des catégories classiques, et la nécessité pour l'ethnologue de passer par un véritable désapprentissage des repères précédemment acquis en se dégageant des systèmes de convention - les "cadres " goffmaniens - ici très instables puisque l'action mise en scène peut à tout moment passer d'une intention ludique à une intention rituelle. Le risque permanent de dérapage porte à un point extrême l'incertitude présidant à toute observation ethnologique d'une performance théâtrale. Nous pouvons dès lors nous demander, avec l'auteur (p.74), si un spectacle qui contient la possibilité de faire advenir une action réelle - qui ne soit plus action jouée - peut être encore qualifié de théâtre. En générant la possibilité d'une rupture de ses propres cadres de convention, le théâtre yoruba révèle son instabilité intrinsèque et témoigne par là même de la «fragilité » d'une supposée « culture yoruba ». L'instabilité artistique du genre traduit l'incertitude sociale et culturelle de son public. 
5 La fiction culturelle à laquelle se réfère le théâtre yoruba plonge ses racines dans l'histoire politique et sociale du projet national autour d'un État yoruba (Yorubaland) toujours virtuel. La longue partie historique qui occupe le cœur du livre, sans doute indispensable et qui passionnera les africanistes, fait perdre un peu de vue l'objet central de ce travail. Mais elle permet de replacer le théâtre traditionnel yoruba dans un processus politique complexe, dont le dernier avatar est l'actuel micro-nationalisme ressurgi dans les années 1980 à la faveur de la crise du modèle fédéraliste nigérian. Comme entité linguistique, les Yoruba se seraient installés au confluent des fleuves Niger et Benoé il y a 3500 ans. L'âge d'or auquel se réfère le théâtre yoruba est celui de l'empire d'Oyo, ville fondée au xi siècle et centre d'un puissant État militaire jusqu'en 1820. Parallèlement, les Yoruba ont une histoire américaine liée à l'esclavage. Au début du XIX ${ }^{e}$ siècle, une partie des descendants d'esclaves envoyés outre-Atlantique furent libérés par les Anglais - en contrepartie de leur fidélité à la couronne britannique dans la guerre d'Indépendance américaine - puis installés en Afrique dans ce qui deviendra la Sierra Leone. De cette population composite émergera une élite lettrée, chrétienne et anglophone qui essaimera dans toute l'Afrique de l'Ouest en propageant l'idée d'une renaissance yoruba ${ }^{4}$. S'est engagé à partir des années 1890 et de la colonisation anglaise ce que Bernard Müller qualifie de "processus de mise en forme historique et de réification politique d'une entité collective», une "greffe» permettant «de reconnaître le statut d'élite indigène à un groupe initialement allogène » (p. 101). Ce nationalisme culturel, dans l'émergence duquel les missions chrétiennes précédant la colonisation britannique jouèrent aussi leur rôle, n'eut rien d'un mouvement populaire, mais fut l'œuvre et l'instrument d'un milieu bourgeois, intellectuel et aisé, celui-là même qui aujourd'hui anime et produit le théâtre traditionnel. Cette petitebourgeoisie, qui soutient également d'autres manifestations - publications, conférences - de cet imaginaire culturel, descend directement de ceux qui au XIX ${ }^{\mathrm{e}}$ siècle ont élaboré grâce à des enquêtes de type ethnographique le corpus de la "culture yoruba", bricolage amalgamant des fragments de récits d'origine variée et se référant à une cosmogonie et à un récit des origines - le corpus d'Ifà - que l'on retrouve évidemment dans le théâtre. Le nationalisme yoruba s'enracine dans le mythe d'une continuité historique de l'unité culturelle et politique yoruba, mythe qui est une reconstitution mémorielle partielle et partiale autour du souvenir du royaume d'Oyo.

6 L'intérêt de ce livre se situe bien au-delà de sa seule dimension monographique: il propose une stimulante réflexion à la fois sur les conditions de production du savoir ethnographique, notamment sur les difficultés de l'immersion de l'anthropologue dans la société qu'il souhaite étudier - ainsi l'auteur avoue-t-il n'avoir " pas compris grandchose » à sa première représentation de théâtre yoruba - et sur le mode de traitement du théâtre, voire de toute manifestation spectaculaire, par les sciences sociales. Ce n'est qu'en rendant compte des situations directement observées sans a priori sur ce que ces situations devraient être si le schéma classique, le cadre orthodoxe, était scrupuleusement respecté par les acteurs que le chercheur peut saisir ce qui se joue effectivement sur la scène et dans le public. En l'occurrence, l'auteur a analysé dans leur actualité les représentations de la pièce Odu-Ifà encore jouées par des troupes de théâtre, même si elles ne correspondaient plus au modèle qu'il avait auparavant en tête, celui de l'âge d'or du genre. Cette observation lui permet de percevoir le théâtre d'aujourd'hui comme une forme expérimentale, s'éloignant encore et toujours de l'ancien Yoruba Theatre, ce qu'il préfère interpréter comme l'annonce d'une ère nouvelle plutôt que comme le signe d'une décadence. En transposant le rituel dans le 
cadre théâtral, Odu-Ifà est une pratique spectaculaire qui reflète les aspirations et les doutes de son public, cette classe moyenne qui cherche à se situer dans une société en restructuration. Le théâtre est de ce fait investi d'un rôle politique fondamental puisqu'il donne l'occasion à la petite-bourgeoisie contemporaine de s'approprier la fiction culturelle yoruba pour conserver ou consolider une certaine hégémonie face aux anciennes élites. Chaque représentation est ainsi une occasion de peser sur des rapports de force, et de remettre en jeu la tradition, pour reprendre la judicieuse formule fournissant son titre au livre.

\section{NOTES}

1. Thèse de doctorat soutenue en janvier 2000 sous la direction de Jean Bazin: Théâtre, nationalisme et travail culturel au Nigeria aujourd'hui. Essai de description d'une pièce de Yoruba Theatre. Paris, EHESS.

2. Howard S. Becker, Les Mondes de l'art. Paris, Champs-Flammarion, 2006 (1re éd. angl. 1982).

3. Le plus illustre auteur de théâtre yoruba étant sans doute le prix Nobel de littérature de 1986, Wole Soyinka.

4. Les Anglais stoppèrent la recherche d'une unité politique yoruba en créant le Nigeria en 1914. Depuis l'Indépendance du pays en 1960, de nombreux soubresauts politiques ont provoqué un morcellement de plus en plus important - douze États fédérés en 1980, trente-six depuis 1996, dont huit yorubaphones.

\section{AUTEURS}

\section{THIERRY BONNOT}

Thierry.Bonnot@ehess.fr 